\title{
Dynamics of Nitrous Oxide Reductase Genes (nos $Z$ ) in Intertidal Rocky Biofilms and Sediments of the Douro River Estuary (Portugal), and their Relation to N-biogeochemistry
}

\author{
C. Magalhães • N. Bano • W. J. Wiebe • A. A. Bordalo • \\ J. T. Hollibaugh
}

Published online: 12 July 2008

(C) Springer Science + Business Media, LLC 2008

Erratum to: Microb Ecol 55(2):259-269

DOI 10.1007/s00248-007-9273-7

In the original version of the article, errors were detected in Table 1 as follows:

1. There was no unit for $\mathrm{N}_{2} \mathrm{O} / \mathrm{N}_{2}$, which is in $\%$.

2. Net $\mathrm{N}_{2} \mathrm{O}$ fluxes for May 2002 is $0.02 \pm 0.01 \mathrm{nmol} / \mathrm{g}$ wet $\mathrm{sed} / \mathrm{h}$ and not $1.08 \pm 0.50 \mathrm{nmol} / \mathrm{g}$ wet sed $/ \mathrm{h}$.

3. $\mathrm{N}_{2} \mathrm{O} / \mathrm{N}_{2}(\%)$ for February, March, July and August is $6.41 \pm 0.50,7.55 \pm 0.24,0.15 \pm 0.01$ and $1.38 \pm 0.17$, respectively and not $6.03 \pm 0.27,7.02 \pm 0.12,0.65 \pm$ 0.03 and $0.14 \pm 0.03$, respectively.

The correct Table 1 is given here.

The online version of the original article can be found at http://dx.doi. org/10.1007/s00248-007-9273-7.

C. Magalhães $(\square) \cdot$ A. A. Bordalo

Laboratory of Hydrobiology, Institute of Biomedical Sciences,

University of Porto,

Largo Professor Abel Salazar, No 2,

4099-003 Porto, Portugal

e-mail: c_magalhaes@yahoo.com

C. Magalhães · A. A. Bordalo

Centre of Marine and Environmental Research,

Rua dos Bragas, no 289,

4050-123 Porto, Portugal

N. Bano $\cdot$ W. J. Wiebe $\cdot$ J. T. Hollibaugh

Department of Marine Sciences, University of Georgia,

Athens, GA 30602, USA 
Table 1 Sediment net $\mathrm{N}_{2}$ and $\mathrm{N}_{2} \mathrm{O}$ fluxes, $\mathrm{N}_{2} \mathrm{O} / \mathrm{N}_{2}$ ratios, and water column $\mathrm{NO}_{3}^{-}+\mathrm{NO}_{2}^{-}$concentrations and salinity during the monthly sampling survey at the $\mathrm{SZ}$ site (mean $\pm \mathrm{SDV}$ of three replicates)

\begin{tabular}{|c|c|c|c|c|c|}
\hline & $\begin{array}{l}\text { Net } \mathrm{N}_{2} \text { Fluxes } \\
\left(\mathrm{nmol} \mathrm{weight}^{-1} \mathrm{~h}^{-1}\right)\end{array}$ & $\begin{array}{l}\text { Net } \mathrm{N}_{2} \mathrm{O} \text { fluxes } \\
\text { (nmol weight }^{-1} \mathrm{~h}^{-1} \text { ) }\end{array}$ & $\begin{array}{l}\mathrm{N}_{2} \mathrm{O} / \mathrm{N}_{2} \\
(\%)\end{array}$ & $\begin{array}{l}\mathrm{NO}_{3}^{-}+\mathrm{NO}_{2}^{-} \\
(\mu \mathrm{M})\end{array}$ & $\begin{array}{l}\text { Salinity } \\
\text { (psu) }\end{array}$ \\
\hline Feb 2002 & $2.81 \pm 0.21$ & $0.19 \pm 0.02$ & $6.41 \pm 0.50$ & $34.5 \pm 1.1$ & 22.7 \\
\hline Mar 2002 & $2.62 \pm 0.05$ & $0.20 \pm 0.06$ & $7.55 \pm 0.24$ & $31.9 \pm 0.9$ & 15.1 \\
\hline Apr 2002 & $3.09 \pm 0.31$ & $0.03 \pm 0.03$ & $1.11 \pm 0.10$ & $39.6 \pm 0.3$ & 16.0 \\
\hline May 2002 & $3.07 \pm 0.05$ & $0.02 \pm 0.01$ & $0.66 \pm 0.03$ & $21.5 \pm 0.1$ & 24.0 \\
\hline Jun 2002 & $0.96 \pm 0.02$ & $0.01 \pm 0.00$ & $0.65 \pm 0.03$ & $5.2 \pm 0.3$ & 26.8 \\
\hline July 2002 & $0.74 \pm 0.04$ & $0.00 \pm 0.00$ & $0.15 \pm 0.01$ & $12.8 \pm 0.2$ & 25.0 \\
\hline Aug 2002 & $6.17 \pm 0.47$ & $0.08 \pm 0.04$ & $1.38 \pm 0.17$ & $65.0 \pm 0.6$ & 13.9 \\
\hline Sep 2002 & $3.05 \pm 0.18$ & $0.02 \pm 0.02$ & $0.78 \pm 0.06$ & $36.0 \pm 0.5$ & 13.2 \\
\hline Oct 2002 & $0.85 \pm 0.04$ & $0.01 \pm 0.01$ & $0.78 \pm 0.06$ & $20.6 \pm 1.1$ & 22.7 \\
\hline Nov 2002 & $4.41 \pm 0.33$ & $0.01 \pm 0.01$ & $0.30 \pm 0.00$ & $59.1 \pm 1.8$ & 9.0 \\
\hline Dec 2002 & $8.36 \pm 0.57$ & $0.02 \pm 0.02$ & $0.24 \pm 0.03$ & $59.9 \pm 1.0$ & 3.4 \\
\hline Jan 2003 & $8.75 \pm 0.39$ & $0.13 \pm 0.13$ & $1.48 \pm 0.07$ & $92.0 \pm 2.8$ & 1.5 \\
\hline
\end{tabular}

Data from Magalhães et al. [18]. 